\title{
The Development of Power Amplifier for the High-Power Magnetic Suspension Bearing Blower
}

\author{
Yu Wentao ${ }^{1, a}$, Li Hongwei ${ }^{1, b}$, Liu Shuqin ${ }^{1, c, *}$ \\ ${ }^{1}$ Shandong University, Jingshi Road 17923, Jinan city, Shandong province, China \\ awintop@sdu.edu.cn, ' 1 hw08@sdu.edu.cn, 'Ishuqin@sdu.edu.cn \\ * Corresponding author. Liu Shuqin Tel.: +86-0531-81696186; fax: +86-0531-88399385.
}

Keywords: AMB, FPGA, Three-Level Power Amplifier, the Blower

\begin{abstract}
The blower with Active Magnetic Bearing (AMB) has the advantage of high speed, high reliability, small size, good adaptability. Power amplifier is an important part of the magnetic bearing system, directly determines the magnetic bearing system performance. The three-level PWM signal generation algorithm is analyses in this paper, and the IR2113 gate driven circuits with a negative bias is designed, and build a three-level magnetic bearing power amplifier. The gate driven signal of circuit is test, and entire power amplifier tracking step signal and sinusoidal signal also be test, the test results indicate that the three-level power amplifier meet the design requirements. At last, the power amplifier application on the blowers with AMB successful.
\end{abstract}

\section{Introduction}

Active Magnetic Bearings (AMB) using electromagnetic force to suspend rotor, with no friction loss, no lubrication, high-speed, non-polluting characteristics. ${ }^{[1]}$ The blower with AMB has the advantage of high speed, high reliability, small size, good adaptability ${ }^{[2]}$. Power amplifier is an important part of the overall magnetic bearing system, directly determines the magnetic bearing system performance ${ }^{[3]-[7]}$. Depending on the realization of the principle, magnetic bearings power amplifier divided into linear power amplifier and switching power amplifier. With respect to the linear power amplifier, switching power amplifier has the advantage of low power consumption and high conversion efficiency; occupy a dominant position in large power amplifiers. Switching power amplifier can be divided into two-level amplifier switching amplifier and three-level switching amplifier depending on the switching signal. In two-level switching amplifier, the magnitude of current ripple is related to the DC voltage. Increases the DC voltage to improve the dynamic characteristics of the system, it will increase the electromagnetic coil current ripple at the same time. The current ripple of three-level switching power amplifier is not only smaller than the two-level power amplifier, but also nothing affected by the DC voltage ${ }^{[4]-[7]}$, and therefore more suitable for high-power active magnetic bearings. In this paper, using FPGA chip to generate three-level switching signals, half-bridge circuit using IGBT modules and IGBT gate drive circuit is designed based on the drive requirements.

\section{The Three-level PWM Signal Generation Algorithm:}

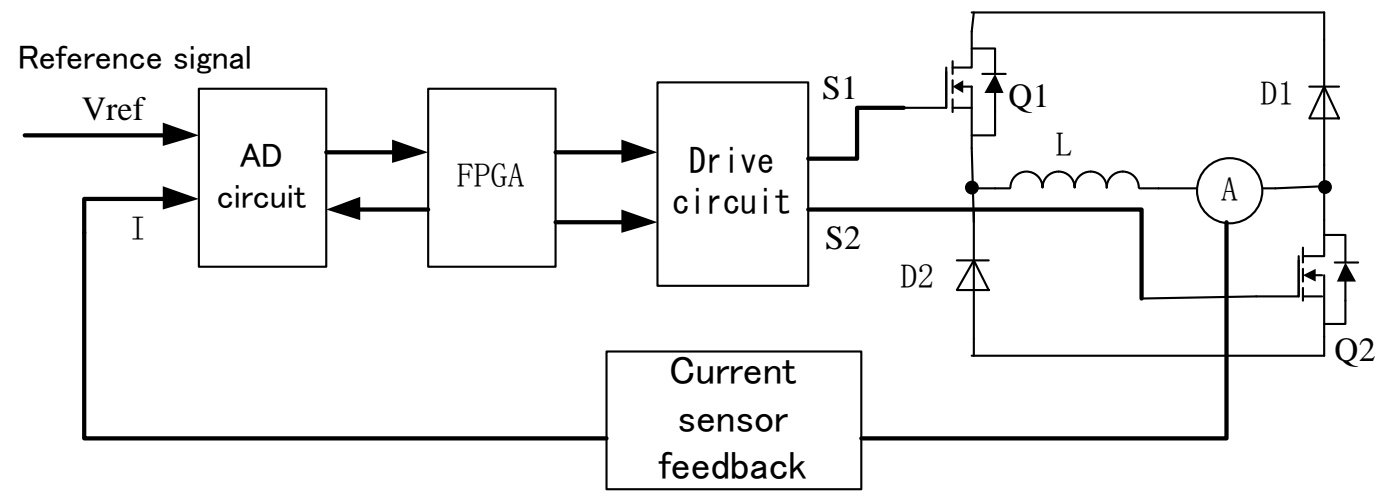

Figure 1 Three-level Amplifier Main Circuit Topology 
Figure 1 is the main circuit topology for three-level amplifier. Vref is reference signal inputted to the power amplifier from the controller, I is the magnetic bearing coil current. FPGA obtain the reference signal Vref and the coil current feedback value I through AD circuit. The driving signals S1 and S2 for the main half-bridge circuit upper arm and lower arm IGBT is generated by FPGA chip through three-level algorithm, and transported to the drive circuit amplifies to drive IGBT modules of half-bridge circuit, in order to produce the inductor coil current. FPGA chip uses the XILINX Company's XC3S500, which has 500,000 logic units and 232 freely configurable I / O ports, a single chip will be able to complete the three-level PWM signal for all the power amplifier of AMB.

With different two-level signal, three-level signal has not only the current states of increase and decrease, but also more of a freewheeling current state that the coil current is basically unchanged. To illustrate the principles of the three-level, the introduction of the switching function $\mathrm{Si}(\mathrm{i}=1 \sim 2)$

$$
S_{\mathrm{i}}=\left\{\begin{array}{lll}
1, & Q_{\mathrm{i}} & \text { on } \\
0, & Q_{\mathrm{i}} & \text { off }
\end{array}\right.
$$

For the half-bridge circuit, the switching function of Q1 and Q2 is satisfied the following equation:

$$
\begin{gathered}
S_{1}= \begin{cases}1, & \text { 当 } 0.5 U_{T \text { max }}+\Delta U_{C}(t)<U_{T}(t) \\
0, & \text { 当 } 0.5 U_{T \text { max }}+\Delta U_{C}(t)>U_{T}(t)\end{cases} \\
S_{2}= \begin{cases}1, & \text { 当 } 0.5 U_{\mathrm{T} \text { max }}-\Delta U_{C}(t)>U_{\mathrm{T}}(t) \\
0, & \text { 当 } 0.5 U_{\mathrm{T} \text { max }}-\Delta U_{C}(t)<U_{\mathrm{T}}(t)\end{cases}
\end{gathered}
$$

Where: $\mathrm{U}_{\text {Tmax }}$ is the maximum value of the triangular carrier signal $\triangle \mathrm{U}_{\mathrm{C}(\mathrm{t})}$ is input error control signal, $\triangle \mathrm{U}_{\mathrm{C}(\mathrm{t})}=$ Vref $-\mathrm{I}$ $\mathrm{U}_{\mathrm{T}(\mathrm{t})}$ is triangular carrier signal

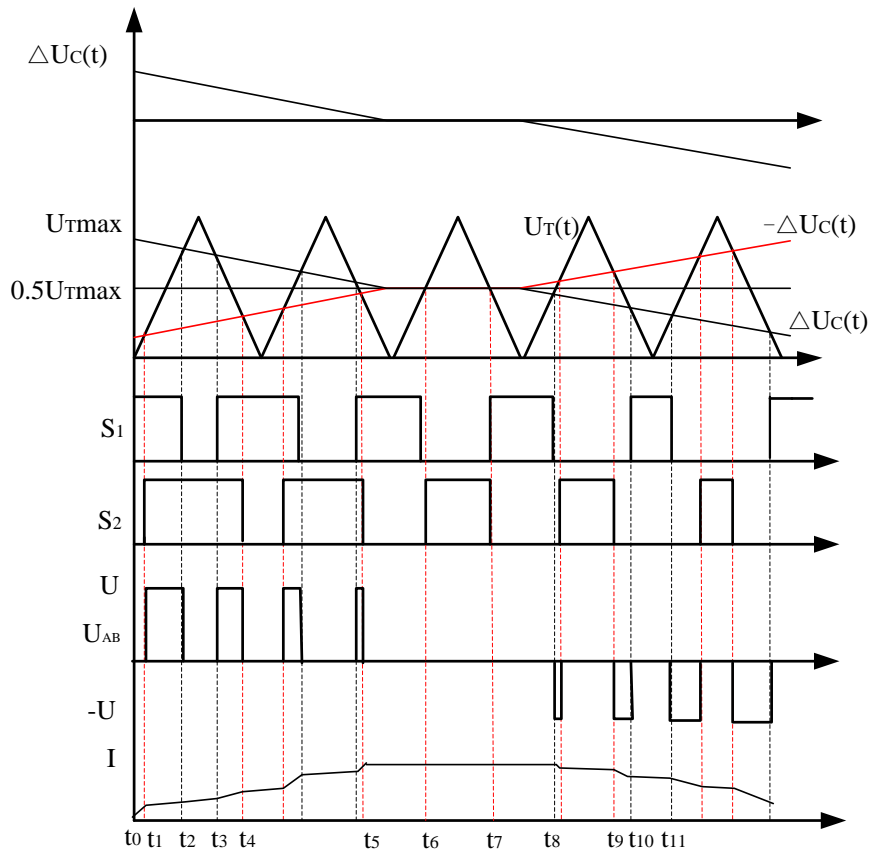

Figure 2 three-level PWM pulse generator schematic

Figure 2 is the three-level PWM generation principle diagram. When the error signal $\triangle \mathrm{Uc}(\mathrm{t})$ is greater than zero, it will produce greater than $50 \%$ duty cycle corresponding pulse signal according to the switching function, Upper and lower bridge arms of half-bridge circuit are turned on simultaneously, the coil current is increased. And the bigger the error, the greater the duty cycle, the current rise faster. When the error becomes smaller, the duty cycle becomes smaller; the current 
rise speed is slow. When the error signal is equal to 0, the pulse signals S1 and S2 are 50\% duty cycle, No load bus voltage across the coil, the coil current is only freewheeling state, so the current is substantially unchanged. When the error signal is less than zero, the duty cycle of the generated pulse signal are less than 50\%, Upper and lower arm of half-bridge circuit are turned off simultaneously, The freewheeling diode D1 and D2 are turn on at the same time, coil current is reduced. And the greater the error of between control signal and coil current, the faster the coil current.

In summary, the three-level power amplifier of AMB works at this several states, which can let the current in the coil followed the control signal increases, decreases or freewheeling.

\section{IGBT Gate Driven Circuit Designed}

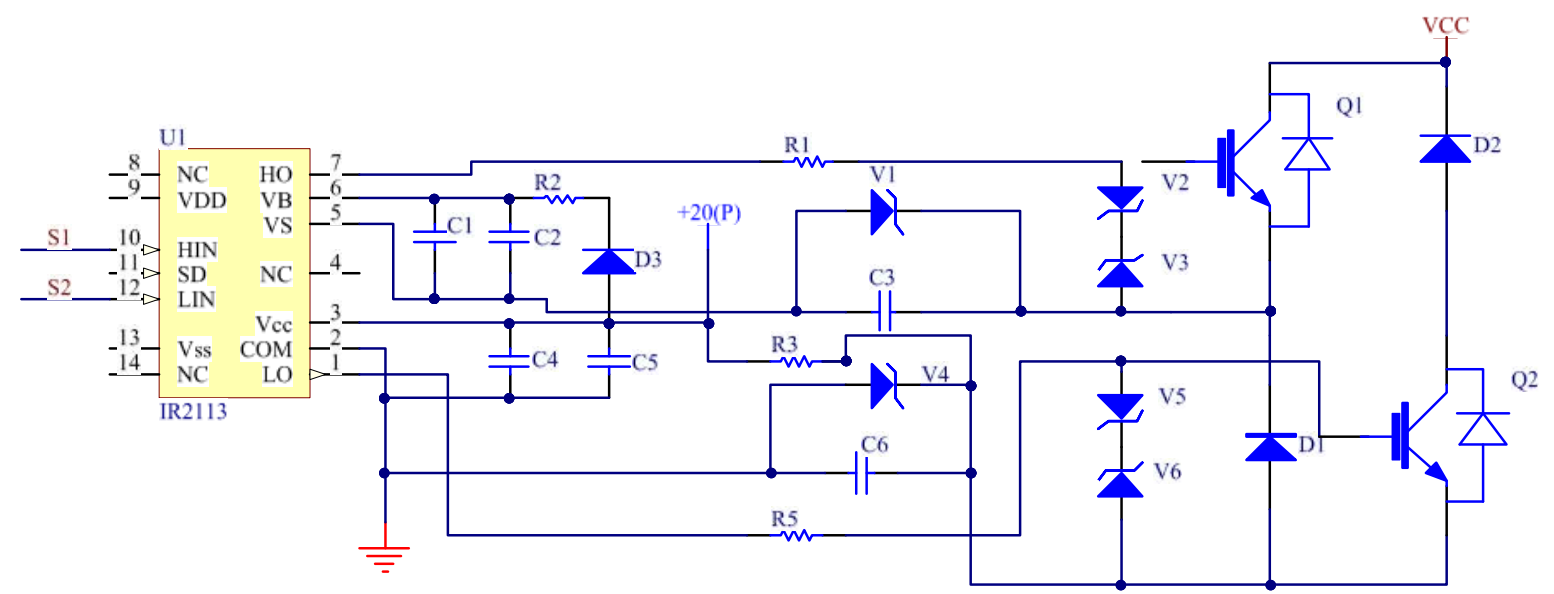

Figure 3 the schematic of IGBT gate driven circuit

Half-bridge main circuit using IGBT module FR25R12KE3, It is specifically designed for converter power module, the technical parameters is: maximum pressure is $1200 \mathrm{~V}$, rated current is 25A, Since the module has seven IGBT and one power diode, two half-bridge circuit can be designed, the merits of IGBT gate drive circuit design is directly related to long-term operation of the IGBT system reliability. The positive gate voltage should be enough to make IGBT turn on fully, and keep the minimum on-state resistance, When IGBT need to be turned off, in order to ensure that IGBT remains off even if the du/dt noise of collector emitter voltage disturbing, A reverse bias voltage must be applied on the gate electrode, it also can reduce the loss of IGBT turn off. IR2113 is used as the gate drive circuit in this paper. The IR2113 is a power switch device driver ASIC with dual channel high voltage, high speed voltage type, bootstrap floating power supply, simple drive circuit, and only one chip can drive the upper and lower arm of bridge circuit to turn on or off at the same time, But its shortcoming is: it can’t generate negative voltage, and anti-jamming weakly. In order to overcome this shortcoming, the figure 3 circuit is designed in this paper to realize the negative voltage on drive circuit. The circuit principle is as follows: When the circuit is powered on, the $20 \mathrm{~V}$ power supply charges capacitor C6 through R3, and C6 will keep 5V voltage under the effect of the zener diode V4, so the source voltage of IGBT is 5V. When the LIN of IR2113 is inputted high level, the LO of IR2113 will output high level voltage of 20V, then the voltage between the gate and the source of the lower IGBT Q2 is $15 \mathrm{~V}$, and the IGBT turns on normally. When the LIN is inputted low level, the LO will output 0 voltage, then the voltage between the gate and the source of the lower IGBT Q2 is -5V. Similarly, the capacitor C3 has 5V voltage, so when the HIN input high level and low level, the driving voltage between the gate and the source of the upper IGBT Q1 are respectively 15V and -5V.

\section{Experimental Verification}

In this paper, the three-level power amplifier is designed based on the control strategy and developed IGBT gate driven circuit. 
First, the IGBT gate driven circuit is test if it meets the design requirement. The LIN pin and LO pin of IR2113 are measured shown in Fig 3, the switching frequency is $20 \mathrm{KHz}$, and the results shown in Fig 4, in which the above signal is LIN, and the lower signal is LO, the LO signal is the voltage between gate and source of IGBT. From Fig.4, we can see the LO signal is 15V when the LIN is high level and is $-5 \mathrm{~V}$ when the LIN is lower signal. So the gate driven circuit meets the driven requirement of IGBT, reduces the switching losses and improve the IR2113 chip noise immunity.

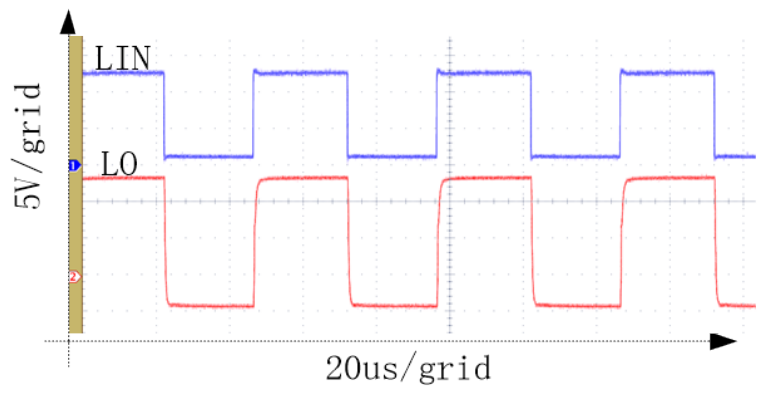

Figure 4 the test result of gate driven circuit of IGBT

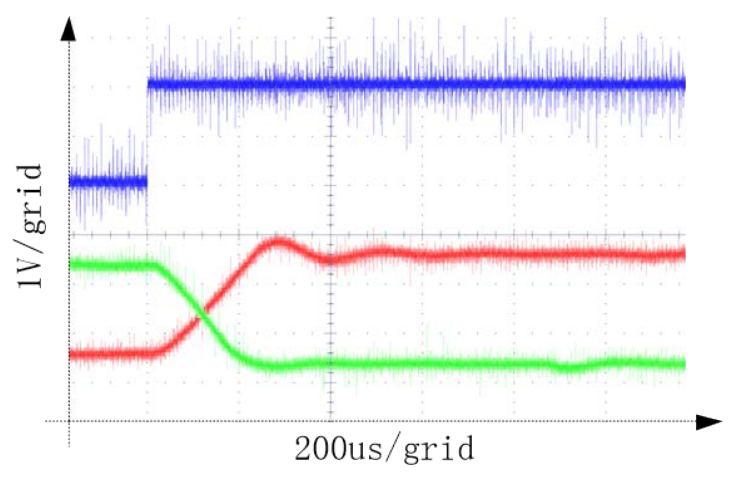

(a) the Step Signal Tracking Test Figure 5 Test of the Upper and Lower Coils Current Track Control signal.

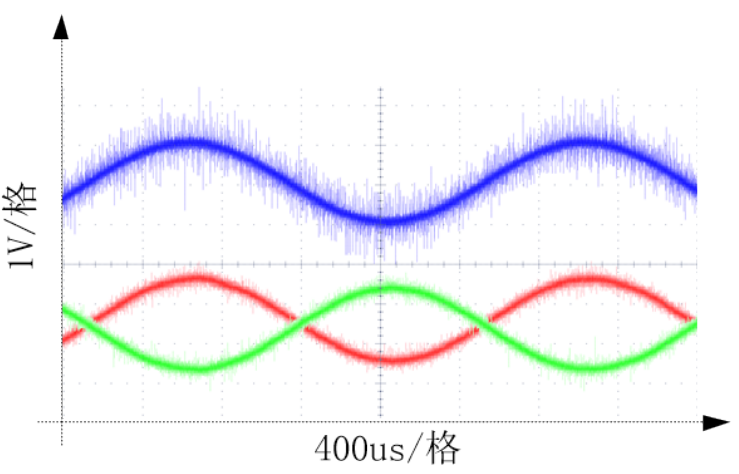

(b) the Sinusoidal Signal Tracking Test

Figure 5 is schematic diagram of the upper and lower magnetic bearing coils current track the control signal. In order to improve the dynamic characteristics of magnetic bearing, the current signal of the upper and lower coils is usually inverse relationship. Figure 5(a) is the step signal tracking test. Test conditions: bus voltage is $150 \mathrm{~V}$, magnetic bearing magnetic coil resistance is 1.5 $\Omega$, coil inductance is about $20 \mathrm{mH}$. Step signal is $2 \mathrm{~V}$, step current generated in the coils are 2A.Form figure 5(a), and we can learned that the time of the coil current track changes in the speed of the signal is about 400us time. Figure 5 (b) is a sinusoidal signal tracking test, a sinusoidal input signal frequency is $500 \mathrm{~Hz}$, Test conditions are the same with above, the frequency of input sinusoidal signal is $500 \mathrm{~Hz}$. It can be seen that the coil current signal can be tracked with sinusoidal signal very well.

The power amplifier is applied in the maglev blower, the rotor can be suspended stabilize in less than $30 \mathrm{~ms}$ and the blower can work stable at $250 \mathrm{~Hz}$ or more, so the power amplifier meet the design requirements.

\section{Conclusion}

The three-level PWM signal generation algorithm is analyses in this paper, and the IR2113 gate drive circuits with a negative bias is designed, and build a three-level magnetic bearing power amplifier. Then the gate driven signal of circuit is test, and entire power amplifier tracking step 
signal and sinusoidal signal also be test, The test results may indicate that the designed three-level power amplifier meet the design requirements.

\section{Acknowledgements}

This research work was financially supported by Natural Science Foundation of China (11302120), Natural Science Foundation of Shandong Province (ZR2011EEQ029) and (ZR2012EEM031), Ji'nan International Cooperation Project (201305057).

\section{References}

[1] G Schweitzer, H Bleuler, A Traxler, Active Magnetic Bearings, ETH, 1994

[2] Xie Youbai, Yuan Chongjun, Yu Lie, Wang Xiping, An Application of Active Magnetic Bearings in a Mixed Gas Blower, Proc.of 4th International Symposium on Magnetic Bearings, Aug 23-26, 1994: 499 504

[3] Wentao Yu, Youpeng Fan, Shuqin Liu, Deguang Li, Research on MATLAB Simulation of Three Level Power Amplifier for Magnetic Bearings, APPEEC 2010

[4] ZHANG Liang, FANG Jiancheng, Study on Three-level PWM Switching Power Amplifier for Active Magnetic Bearing biased with Permanent Magnet, POWER ELECTRONICS, 2006/2 1-2

[5] ZHANG Dan-hong DONG Rui, Design of the Tristate Power Amplifier for Active Magnetic Bearings Control, POWER ELECTRONICS, 2006/6 112-114

[6] YU Wen-tao LIU Shu-qin, Design of Protection Circuit for Current-mode Power Amplifier, POWER ELECTRONICS, 2007 /41 /07, 89-91

[7] CHANG Xiao XU Longxiang DONG Jiyong, Digital Power Amplifier of Active Magnetic Bearing, JOURNAL OF MECHANICAL ENGINEERING, 2010 46(20), 9-14 